\title{
Modified Green's Functions for Shallow Water Acoustic Wave Propagation
}

\author{
J.A.F. Santiago ${ }^{\mathrm{a}}$ and L.C. Wrobel ${ }^{\mathrm{b}}$ \\ ${ }^{a}$ COPPE/UFRJ, Programa de Engenharia Civil, Rio de Janeiro, Brazil \\ ${ }^{\mathrm{b}}$ Brunel University, Department of Mechanical Engineering, Uxbridge UB8 3PH, UK
}

\begin{abstract}
This article presents an assessment of alternative forms of the Green's function for boundary element simulations of acoustic wave propagation in shallow water. It is assumed that the problem is two-dimensional, the source of acoustic disturbance is time-harmonic, the velocity of sound is constant and the medium in the absence of perturbations is quiescent.

Efficient implementations of the boundary element method for underwater acoustics should employ Green's functions which directly satisfy the boundary conditions on the free surface and the horizontal parts of the bottom boundary. In the present work, these Green's functions are constructed by using different techniques, namely the method of images, eigenfunction expansions and the Ewald's method.
\end{abstract}

Keywords: boundary element method, shallow water acoustics, Ewald's method, eigenfunction expansions

\section{Introduction}

Increasing concern for coastal areas has, in recent years, focussed studies of ocean acoustic wave propagation on shallow water environments. The most common numerical techniques used to model underwater acoustic wave propagation are ray methods, normal mode methods and parabolic equation methods [1]. The boundary element method (BEM) can also be employed as an alternative technique, particularly for wave propagation over irregular bottom topography, in the frequency domain [2-6].

Since the acoustic domain is bounded by two reflecting surfaces (the ocean bottom and its free surface), efficient BEM implementations for underwater acoustics should employ Green's functions which satisfy the boundary conditions both on the free surface and the horizontal parts of the bottom boundary. These modified Green's functions may be constructed using the method of images, but this leads to very slowly convergent series [3-6].

A popular alternative to improve the convergence of the series is to construct a Green's function in the form of eigenfunction expansions, the so-called normal mode solution. The normal mode solution is also the sum of an infinite number of terms; however, if the evanescent modes are ignored and only the propagating modes are retained, the number of terms in the series becomes finite [3]. In spite of that, convergence problems still remain when the source and field points are located along the same vertical line [2-5].

Recent papers by Linton [7, 8] and Papanicolaou [9] discuss numerous mathematical techniques for accelerating slowly convergent series. They show that one powerful technique is the method of Ewald [10], which is capable of providing dramatic improvements in the speed of convergence. This method has been successfully implemented in the BEM context by Venakides et al. [11], for the calculation of electromagnetic scattering of photonic crystals.

In the present article, a two-dimensional model is studied as representative of coastal regions, which have little variation in the long shore direction. The Ewald's method was derived 
and implemented for speeding-up the calculations of the eigenfunction expansion of the Green's function. The convergence properties, efficiency and accuracy of the different forms of the Green's function obtained by all the above techniques are compared with respect to the number of iterations, particularly close to singularities. The comparison accounts for source points located at a fixed position and field points moving along different vertical and horizontal lines.

\section{Governing equations of the problem}

Consider the problem of acoustic wave propagation in a region $\Omega$ of infinite extent with irregular seabed topography $[4,5]$, shown in Figure 1.

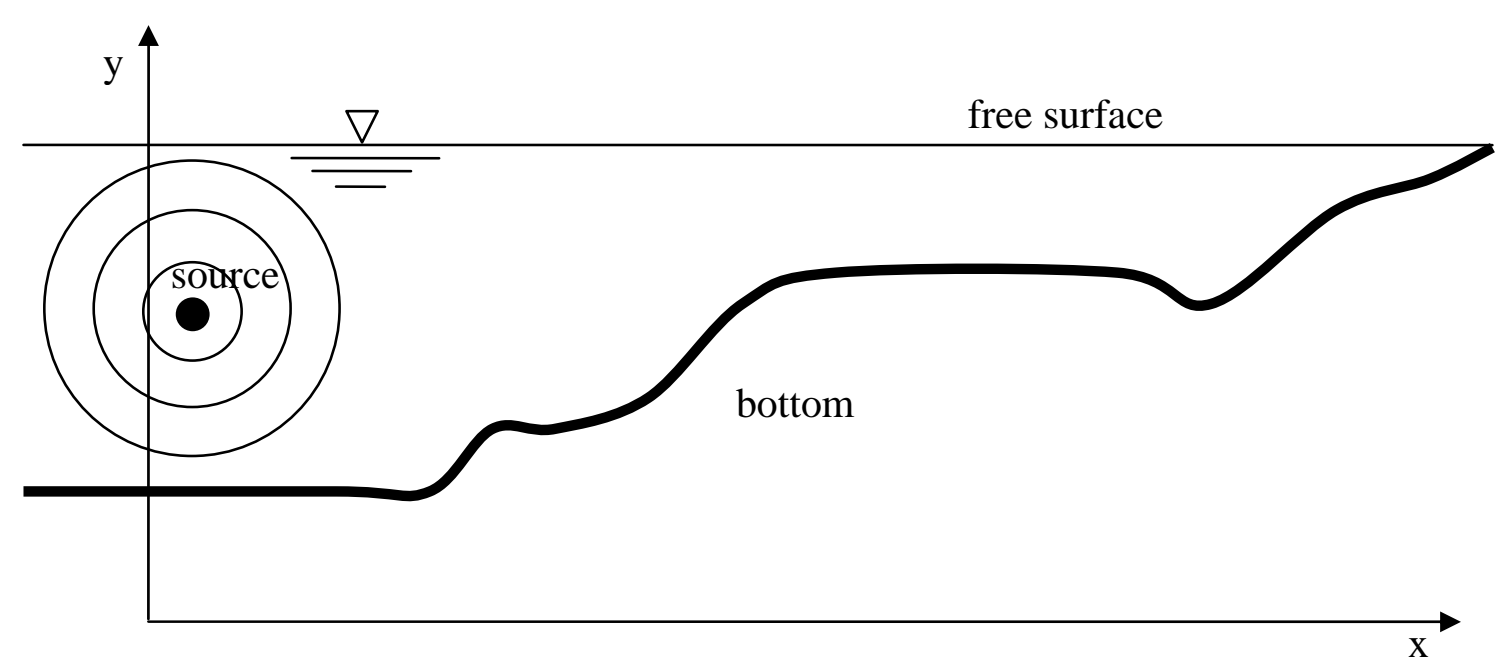

Figure 1: General ocean section for two-dimensional acoustic propagation problems in shallow water

If the medium in the absence of perturbations is quiescent, the velocity of sound is constant and the source of acoustic disturbance is time-harmonic, the problem is governed by the Helmholtz equation [12]

$$
\nabla^{2} \varphi+k^{2} \varphi=-\sum_{\alpha=1}^{N e s} B_{\alpha} \delta\left(\mathbf{E}_{\alpha}, \mathbf{S}\right) \quad \text { in region } \Omega
$$

where $\varphi$ is the velocity potential, $B_{\alpha}$ is the magnitude of the sound source $\mathbf{E}_{\alpha}$ located at $\left(x_{e_{\alpha}}, y_{e_{\alpha}}\right), \mathbf{S}$ is the source point, located at $(u, v)$, Nes is the number of sound sources, $\delta\left(\mathbf{E}_{\alpha}, \mathbf{S}\right)$ is the Dirac delta generalised function and $k=\omega / c$ is the wave number, with $\omega$ the natural frequency and $c$ the velocity of sound in the medium.

The problem is subject to the following boundary conditions:

- at the free surface $\left(\Gamma_{F}\right)$ :

$$
\varphi(\mathbf{X})=0
$$

- at the bottom $\left(\Gamma_{B}\right)$ : 


$$
\frac{\partial \varphi}{\partial n}(\mathbf{X})=0
$$

- Sommerfeld radiation condition at infinity :

$$
\frac{\partial \varphi}{\partial n}(\mathbf{X})=i k \varphi(\mathbf{X})
$$

in which $\mathbf{X}$ is the field point, located at $(x, y), n$ is the outward normal and $i=\sqrt{-1}$.

According to Green's second identity, Equation (1) can be transformed into the following boundary integral equation [13]

$$
C(\mathbf{S}) \varphi(\mathbf{S})=\int_{\Gamma} G(\mathbf{S}, \mathbf{X}) \frac{\partial \varphi}{\partial n}(\mathbf{X}) d \Gamma-\int_{\Gamma} \frac{\partial G}{\partial n}(\mathbf{S}, \mathbf{X}) \varphi(\mathbf{X}) d \Gamma+\sum_{\alpha=1}^{N e s} B_{\alpha} G\left(\mathbf{E}_{\alpha}, \mathbf{S}\right)
$$

where $\Gamma$ is equal to $\Gamma_{F} \cup \Gamma_{B}$ and $G(\mathbf{S}, \mathbf{X})$ is the Green's function. The function $\partial \varphi / \partial n(\mathbf{X})$ represents the normal derivative of the velocity potential. The coefficient $C(\mathbf{S})$ depends on the boundary geometry at the source point $\mathbf{S}$. It is noted that the Green's function implicitly satisfies the Sommerfeld condition, therefore no discretization of the boundary at infinity is necessary.

Instead of using the Green's function $G(\mathbf{S}, \mathbf{X})$ of the Helmholtz equation for a line source in a plane geometry, it is possible to adopt Green's functions which directly satisfy the boundary conditions on $\Gamma_{F}$ and horizontal parts of $\Gamma_{B}$. Therefore, only the irregular parts of the bottom boundary need to be discretized.

\section{Modified Green's functions}

The Green's functions $G(\mathbf{S}, \mathbf{X})$ for the problem mentioned in the previous section were developed by two different means. The first was the image method using multiple source point reflections, while the second used a series of eigenfunctions (normal modes) of the depthseparated equation. An alternative form of the latter was also developed using Ewald's method.

\section{1- Image method}

Constructing a Green's function through the image method, using multiple source point reflections, leads to an infinite series which directly satisfies both boundary conditions, at the ocean bottom and free surface. However, the truncation of the series will lead to the exact satisfaction of only one boundary condition and the approximate satisfaction of the other. Therefore, two types of truncated series can be constructed.

The first type, the modified Green's function $G_{F}(\mathbf{S}, \mathbf{X})$, exactly satisfies the boundary condition at the free surface, but its normal derivative produces a small non-zero value at the bottom boundary:

$$
\begin{aligned}
& G_{F}(\mathbf{S}, \mathbf{X})=i \frac{1}{4}\left\{H_{0}^{(1)}(k r)-H_{0}^{(1)}\left(k r^{(1 F)}\right)+\sum_{m=1}^{\infty} G_{F m}(\mathbf{S}, \mathbf{X})\right\} \\
& G_{F m}(\mathbf{S}, \mathbf{X})=(-1)^{m+1}\left[H_{0}^{(1)}\left(k r_{m}^{(2 F)}\right)-H_{0}^{(1)}\left(k r_{m}^{(3 F)}\right)-H_{0}^{(1)}\left(k r_{m}^{(4 F)}\right)+H_{0}^{(1)}\left(k r_{m}^{(5 F)}\right)\right]
\end{aligned}
$$


The second type, the modified Green's function $G_{B}(\mathbf{S}, \mathbf{X})$, exactly satisfies the boundary condition at the bottom, but the velocity potential produces a small non-zero value at the free surface:

$$
\begin{aligned}
& G_{B}(\mathbf{S}, \mathbf{X})=i \frac{1}{4}\left\{H_{0}^{(1)}(k r)+H_{0}^{(1)}\left(k r^{(1 B)}\right)+\sum_{m=1}^{\infty} G_{B m}(\mathbf{S}, \mathbf{X})\right\} \\
& G_{B m}(\mathbf{S}, \mathbf{X})=(-1)^{m}\left[H_{0}^{(1)}\left(k r_{m}^{(2 B)}\right)+H_{0}^{(1)}\left(k r_{m}^{(3 B)}\right)+H_{0}^{(1)}\left(k r_{m}^{(4 B)}\right)+H_{0}^{(1)}\left(k r_{m}^{(5 B)}\right)\right]
\end{aligned}
$$

where the superscripts $(\mathrm{j} F)$ and $(\mathrm{j} B)$ identify the reflected source points $(\mathrm{j}=1$ to 4$)$.

The distances from the field point $\mathbf{X}$ to the source point $\mathbf{S}$ and its reflections are denoted as $r, r^{(1 F)}, r^{(1 B)}, r_{m}^{(j F)}$ and $r_{m}^{(j B)}$. These distances can be written as:

$$
\begin{aligned}
& r=|\mathbf{X}-\mathbf{S}|=\sqrt{(x-u)^{2}+(y-v)^{2}} \\
& r^{(1 F)}=\left|\mathbf{X}-\mathbf{S}^{(1 F)}\right|=\sqrt{(x-u)^{2}+\left(y-v^{(1 F)}\right)^{2}} \\
& r_{m}^{(j F)}=\left|\mathbf{X}-\mathbf{S}^{(j F)}\right|=\sqrt{(x-u)^{2}+\left(y-v_{m}^{(j F)}\right)^{2}}
\end{aligned}
$$

with:

$$
\begin{aligned}
& v_{m}^{(1 F)}=2 Y_{F}-v \\
& v_{m}^{(2 F)}=-2(m-1) Y_{F}+2 m Y_{B}-v \\
& v_{m}^{(3 F)}=2 m\left(-Y_{F}+Y_{B}\right)+v \\
& v_{m}^{(4 F)}=2 m\left(Y_{F}-Y_{B}\right)+v \\
& v_{m}^{(5 F)}=2(m+1) Y_{F}-2 m Y_{B}-v
\end{aligned}
$$

Equations (6) and (7) are also used for $G_{B}$, but the index $F$ must be replaced by $B$ and vice-versa. In these equations, $Y_{F}$ and $Y_{B}$ are the y co-ordinate of the free surface and bottom, respectively.

\section{2- Eigenfunction expansion}

The Green's function $G_{M}(\mathbf{S}, \mathbf{X})$ exactly satisfies the boundary conditions on the free surface and the bottom boundaries. This second series, in terms of normal modes, can be written as [14]:

$$
G_{M}(\mathbf{S}, \mathbf{X})=\frac{1}{H} \sum_{m=1}^{\infty} G_{M m}(\mathbf{S}, \mathbf{X})
$$




$$
G_{M m}(\mathbf{S}, \mathbf{X})=\sin \left[k_{y m}\left(Y_{F}-v\right)\right] \sin \left[k_{y m}\left(Y_{F}-y\right)\right] \frac{e^{-\sqrt{-k_{x m}}|x-u|}}{\sqrt{-k_{x m}}}
$$

where $H$ is the depth of the free surface $\left(Y_{F}-Y_{B}\right)$. The parameters $k_{x m}$ and $k_{y m}$ are horizontal and vertical wavenumbers, respectively:

$$
\begin{aligned}
& k_{y m}=\left(m-\frac{1}{2}\right) \frac{\pi}{H} \\
& k_{x m}=k^{2}-k_{y m}^{2}
\end{aligned}
$$

\section{3- Ewald's method}

An alternative form of representing Equation (8) is [11]

$$
G_{E}(\mathbf{S}, \mathbf{X})=\frac{2}{H} \sum_{m=1}^{\infty} \sin \left[k_{y m}\left(Y_{F}-v\right)\right] \sin \left[k_{y m}\left(Y_{F}-y\right)\right] \int_{0}^{\infty} \frac{e^{k_{x m} t-\frac{(x-u)^{2}}{4 t}}}{\sqrt{4 \pi t}} d t
$$

The underlying idea of Ewald's representation is to split the integral in Equation (10) into two parts [9],

$$
\begin{aligned}
& G_{E 1}(\mathbf{S}, \mathbf{X})=\frac{2}{H} \int_{0}^{b^{2}} \frac{1}{\sqrt{4 \pi t}} \sum_{m=1}^{\infty} e^{k_{x m} t-\frac{(x-u)^{2}}{4 t}} \sin \left[k_{y m}\left(Y_{F}-v\right)\right] \sin \left[k_{y m}\left(Y_{F}-y\right)\right] d t \\
& G_{E 2}(\mathbf{S}, \mathbf{X})=\frac{2}{H} \sum_{m=1}^{\infty} \sin \left[k_{y m}\left(Y_{F}-v\right)\right] \sin \left[k_{y m}\left(Y_{F}-y\right)\right] \int_{b^{2}}^{\infty} \frac{e^{k_{x m} t-\frac{(x-u)^{2}}{4 t}}}{\sqrt{4 \pi t}} d t
\end{aligned}
$$

where the parameter $b$, which divides the integral in Equation (10), is chosen appropriately, taking account of the position of the source and field points.

In Ewald's approach, the integrals in Equations (11a) and (11b) are manipulated in order to obtain a Green's function involving rapidly decaying series of special functions, such as complementary error and exponential integral functions. Furthermore, the latter function satisfies simple recursive formulae and, hence, is easy to evaluate numerically.

Introducing expressions (9) into Equation (11a) and rearranging gives

$$
G_{E 1}(\mathbf{S}, \mathbf{X})=\frac{2}{H} \int_{0}^{b^{2}} \frac{e^{k^{2} t-\frac{(x-u)^{2}}{4 t}}}{\sqrt{4 \pi t}} \sum_{m=1}^{\infty} e^{-\left(m-\frac{1}{2}\right)^{2} \frac{\pi^{2}}{H^{2}} t} \sin \left[k_{y m}\left(Y_{F}-v\right)\right] \sin \left[k_{y m}\left(Y_{F}-y\right)\right] d t
$$

Using the following identity for theta functions [15], 


$$
\begin{aligned}
& \sum_{m=1}^{\infty} e^{-\left(m-\frac{1}{2}\right)^{2} \frac{\pi^{2}}{H^{2}} t} \sin \left[k_{y m}\left(Y_{F}-v\right)\right] \sin \left[k_{y m}\left(Y_{F}-y\right)\right]= \\
& \frac{H}{4 \sqrt{\pi t}}\left[e^{-\left(\frac{v-y}{2}\right)^{2} t}-e^{-\left(-Y_{F}+\frac{v+y}{2}\right)^{2} t}+\sum_{m=1}^{\infty} \cos (\pi m) \sum_{j=1}^{4}(-1)^{j} e^{\frac{-c_{j m}^{2}}{t}}\right]
\end{aligned}
$$

where the coefficients $c_{j m}(\mathrm{j}=1$ to 4$)$ are of the form:

$$
\begin{aligned}
& c_{1 m}=H m-Y_{F}+\frac{v+y}{2} \\
& c_{2 m}=H m+\frac{v-y}{2} \\
& c_{3 m}=-H m-Y_{F}+\frac{v+y}{2} \\
& c_{4 m}=H m-\frac{v-y}{2}
\end{aligned}
$$

and substituting into Equation (12) gives

$$
G_{E 1}(\mathbf{S}, \mathbf{X})=\frac{1}{4 \pi}\left[\int_{0}^{b^{2}} \frac{e^{k^{2} t-\frac{a_{1}}{t}}-e^{k^{2} t-\frac{a_{2}}{t}}}{t} d t+\sum_{m=1}^{\infty} \cos (\pi m) \sum_{j=1}^{4}(-1)^{j} \int_{0}^{b^{2}} \frac{e^{\frac{-a_{j m}}{t}}}{t} d t\right]
$$

Applying the Taylor expansion of the function $e^{k^{2} t}$ and taking into account that $\int_{0}^{b^{2}} \frac{t^{n}}{t} e^{\frac{-a}{t}} d t=b^{2 n} E_{n+1}\left(\frac{a}{b^{2}}\right)$, Equation (15) becomes:

$$
G_{E 1}(\mathbf{S}, \mathbf{X})=\frac{1}{4 \pi}\left[C_{n}+\sum_{m=1}^{\infty} \cos (\pi m) \sum_{n=0}^{\infty} \frac{k^{2 n} b^{2 n}}{n !} \sum_{j=1}^{4}(-1)^{j} E_{n+1}\left(\frac{a_{j m}}{b^{2}}\right)\right]
$$

where $E_{n+1}$ is the exponential integral function and the coefficients $C_{n}, a_{1}, a_{2}$ and $a_{j m}$ are defined in the form:

$$
\begin{aligned}
& C_{n}=\sum_{n=0}^{\infty} \frac{k^{2 n} b^{2 n}}{n !}\left[E_{n+1}\left(\frac{a_{1}}{b^{2}}\right)-E_{n+1}\left(\frac{a_{2}}{b^{2}}\right)\right] \\
& a_{1}=\frac{(x-u)^{2}+(v+y)^{2}}{4} \\
& a_{2}=\frac{(x-u)^{2}+\left(-Y_{F}+v+y\right)^{2}}{4}
\end{aligned}
$$




$$
\begin{aligned}
& a_{1 m}=\frac{(x-u)^{2}+\left(2 H m-2 Y_{F}+v+y\right)^{2}}{4} \\
& a_{2 m}=\frac{(x-u)^{2}+(2 H m+v-y)^{2}}{4} \\
& a_{3 m}=\frac{(x-u)^{2}+\left(-2 H m-2 Y_{F}+v+y\right)^{2}}{4} \\
& a_{4 m}=\frac{(x-u)^{2}+(2 H m-v+y)^{2}}{4}
\end{aligned}
$$

Equation (11b), in which the interval of integration ranges from $b^{2}$ to $\infty$, can be calculated explicitly in terms of the complementary error function $\operatorname{erfc}(\mathrm{z})$ :

$$
\begin{aligned}
G_{E 2}(\mathbf{S}, \mathbf{X}) & =\frac{2}{H} \sum_{m=1}^{\infty} \sin \left[k_{y m}\left(Y_{F}-v\right)\right] \sin \left[k_{y m}\left(Y_{F}-y\right)\right] \times \\
& \left\{e^{(x-u) \sqrt{-k_{x m}}} \operatorname{erfC}\left[b \sqrt{-k_{x m}}+\frac{(x-u)}{2 b}\right]+e^{-(x-u) \sqrt{-k_{x m}}} \operatorname{erfC}\left[b \sqrt{-k_{x m}}-\frac{(x-u)}{2 b}\right]\right\}
\end{aligned}
$$

Therefore, the final expression of Ewald's representation of the Green's function (8) is:

$$
G_{E}(\mathbf{S}, \mathbf{X})=G_{E 1}(\mathbf{S}, \mathbf{X})+G_{E 2}(\mathbf{S}, \mathbf{X})=\frac{C_{n}}{4 \pi}+\sum_{m=1}^{\infty} G_{E_{m}}(\mathbf{S}, \mathbf{X})
$$

where $G_{E m}(\mathbf{S}, \mathbf{X})$ is given as:

$$
\begin{aligned}
G_{E_{m}}(\mathbf{S}, \mathbf{X})= & \frac{\sin \left[k_{y m}\left(Y_{F}-v\right)\right] \sin \left[k_{y m}\left(Y_{F}-y\right)\right]}{2 H \sqrt{-k_{x m}}} \times \\
& \left\{e^{(x-u) \sqrt{-k_{x m}}} \operatorname{erfc}\left[b \sqrt{-k_{x m}}+\frac{(x-u)}{2 b}\right]+e^{-(x-u) \sqrt{-k_{x m}}} \operatorname{erfc}\left[b \sqrt{-k_{x m}}-\frac{(x-u)}{2 b}\right]\right\}+ \\
& \frac{\cos (\pi m)}{4 \pi} \sum_{n=0}^{\infty} \frac{k^{2 n} b^{2 n}}{n !}\left[\sum_{j=1}^{4}(-1)^{j} E_{n+1}\left(\frac{a_{j m}}{b^{2}}\right)\right]
\end{aligned}
$$

\section{Analysis of coastal regions of constant depth}

\section{1- Example}

A problem of acoustic wave propagation in regions of two different constant depths $(h=$ $2.0 \mathrm{~m}$ and $h=10.0 \mathrm{~m}$ ) was studied in order to compare the performance of the modified Green's 
function representations of the problem. Different situations were considered, in which the position of the source point $\mathbf{S}$ was fixed and the field point $\mathbf{X}$ varied along a vertical and a horizontal line. The sound velocity and frequency are taken to be $1500 \mathrm{~m} / \mathrm{s}$ and $1000 \mathrm{~Hz}$, respectively.

\section{2- Convergence tests}

Figures 2 and 3 present the decay of the real part of the summations of $G_{F_{m}}(\mathbf{S}, \mathbf{X})$, $G_{M_{m}}(\mathbf{S}, \mathbf{X})$ and $G_{E_{m}}(\mathbf{S}, \mathbf{X})$ as a function of the number of terms, for source and field points located at fixed positions along the same horizontal line (figure 2) or the same vertical line (figure 3).

Figure 2 shows that the series in terms of normal modes presents a very good convergence and the series utilising Ewald's method dramatically improves the speed of convergence, with only 4 terms required, except for $b=0.1$ in case (a), which required 9 terms.

When the co-ordinate $x$ of the source and field points is the same, the exponential term of the function $G_{M}(\mathbf{S}, \mathbf{X})$ is equal to one and convergence becomes slow, as can be observed in Figure 3. However, the function $G_{E}(\mathbf{S}, \mathbf{X})$ still presents a very good convergence, with some dependence on the parameter $b$.

Figures 4 and 5 present the number of iterations necessary for the functions $G_{F}(\mathrm{NiGF})$, $G_{B}$ (NiGB), $G_{M}$ (NiGM) and $G_{E}(b)$ to converge, for a source point $\mathbf{S}$ located at the positions $(1.0,8.0) \mathrm{m}$ and $(1.0,5.0) \mathrm{m}$, respectively, and field point $\mathbf{X}$ moving along the same vertical line on which the source point is located, from $y=0.0$ to $y=10.0 \mathrm{~m}$. For the latter function, nine different values for the parameter $b$ were employed.

It can be seen in these figures that the convergence of function $G_{B}$ improved when the field point was placed halfway along the depth. However, a large number of iterations were necessary for the first case with function $G_{B}$, and for both cases with function $G_{F}$, with over 100,000 terms required for convergence at some points. The number of iterations for function $G_{M}$ was around 20,000 in both cases. A much faster convergence was produced by function $G_{E}$, for all values of the parameter $b$. For values of $b$ of 0.05 and 0.1 , the number of iterations was less than 100 for all values of $y$.

Two types of singularities appear in this simple problem of acoustic propagation in a region of constant depth. The first is due to the coincidence of the source and field points at position (1.0,8.0) $\mathrm{m}$ in Figure 4 and (1.0,5.0) $\mathrm{m}$ in Figure 5. The second type of singularity, which appears at position $(1.0,2.0) \mathrm{m}$ in Figure 4, is due to the special function $E_{1}\left(a_{2} / b^{2}\right)$ in Equation (17) going to infinity as the parameter $a_{2}$ in Expression (18b) tend to zero. Function $G_{E}$ with values of $b$ of 0.05 and 0.1 required less than 100 iterations to converge even at the above singular points. 


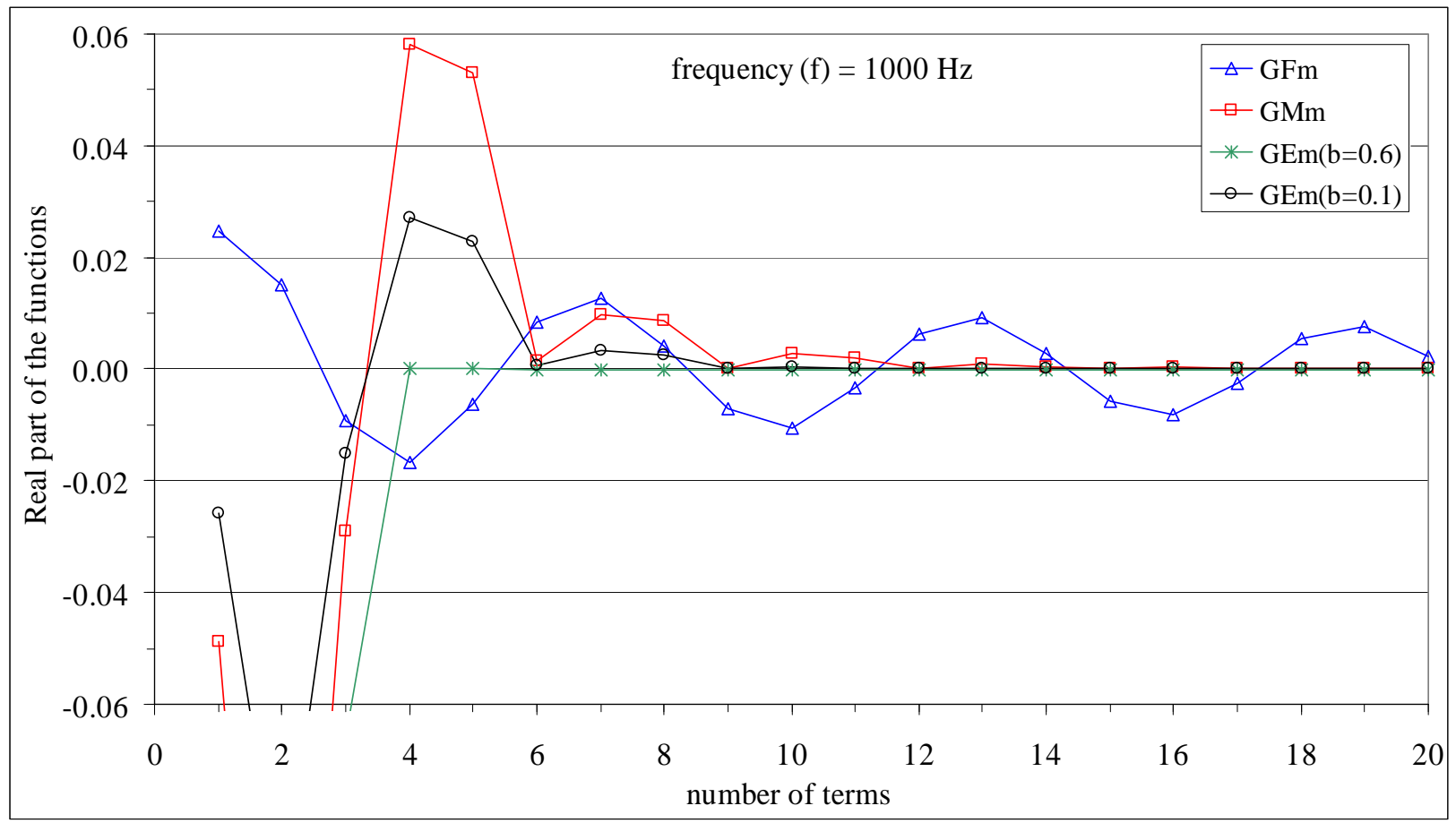

(a) Field point $\mathbf{X}$ close to source point $\mathbf{S}$

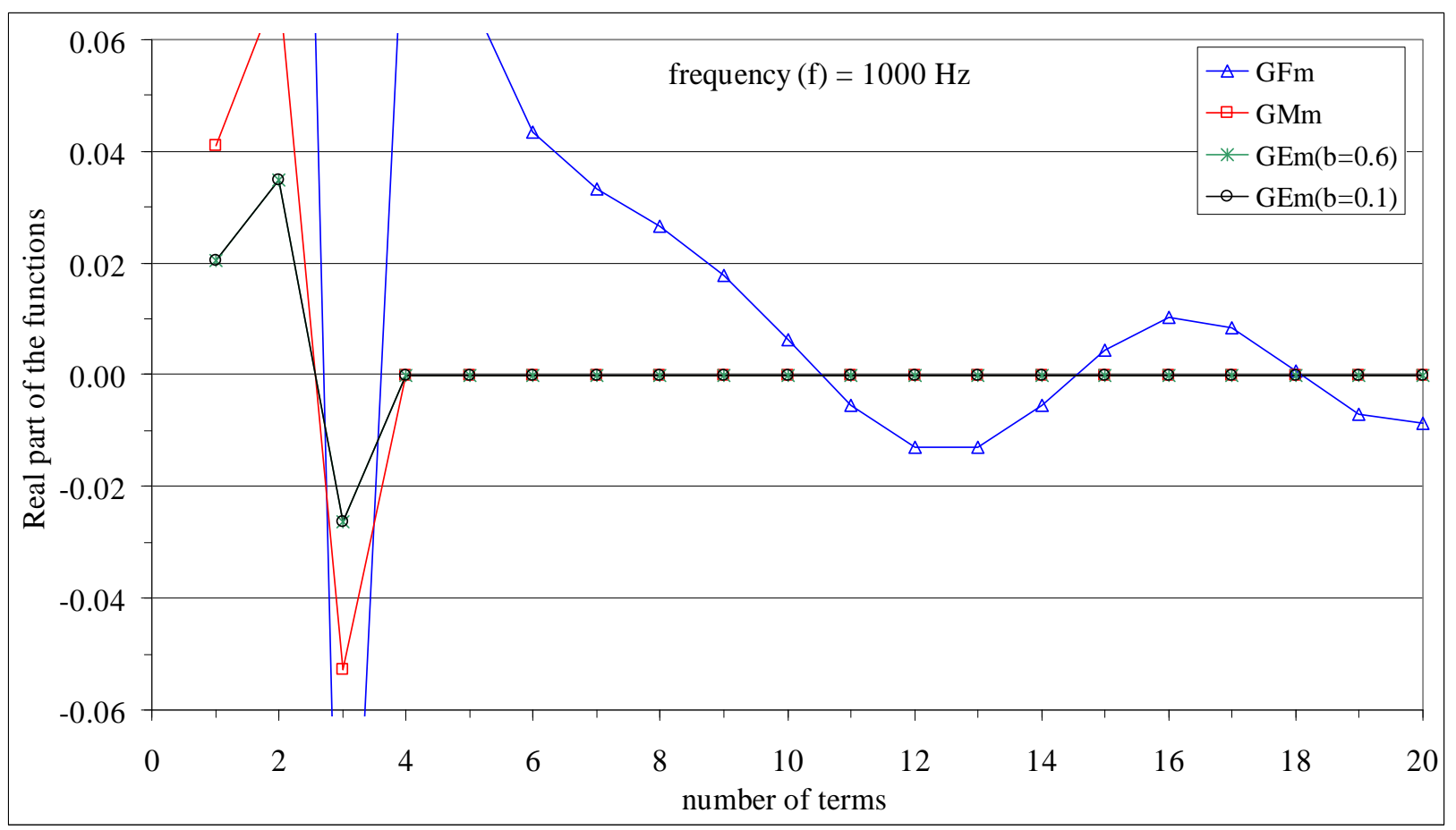

(b) Field point $\mathbf{X}$ far from source point $\mathbf{S}$

Figure 2: Convergence of real part of the summations of $G_{F_{m}}(\mathbf{S}, \mathbf{X}), G_{M_{m}}(\mathbf{S}, \mathbf{X})$ and $G_{E_{m}}(\mathbf{S}, \mathbf{X})$ with number of terms, for $\mathbf{S}$ located at $(1.0,1.3) \mathrm{m}$ and (a) $\mathbf{X}$ located at $(1.2,1.3)$ or (b) $\mathbf{X}$ located at $(10.0,1.3) \mathrm{m}(h=2 \mathrm{~m})$. 


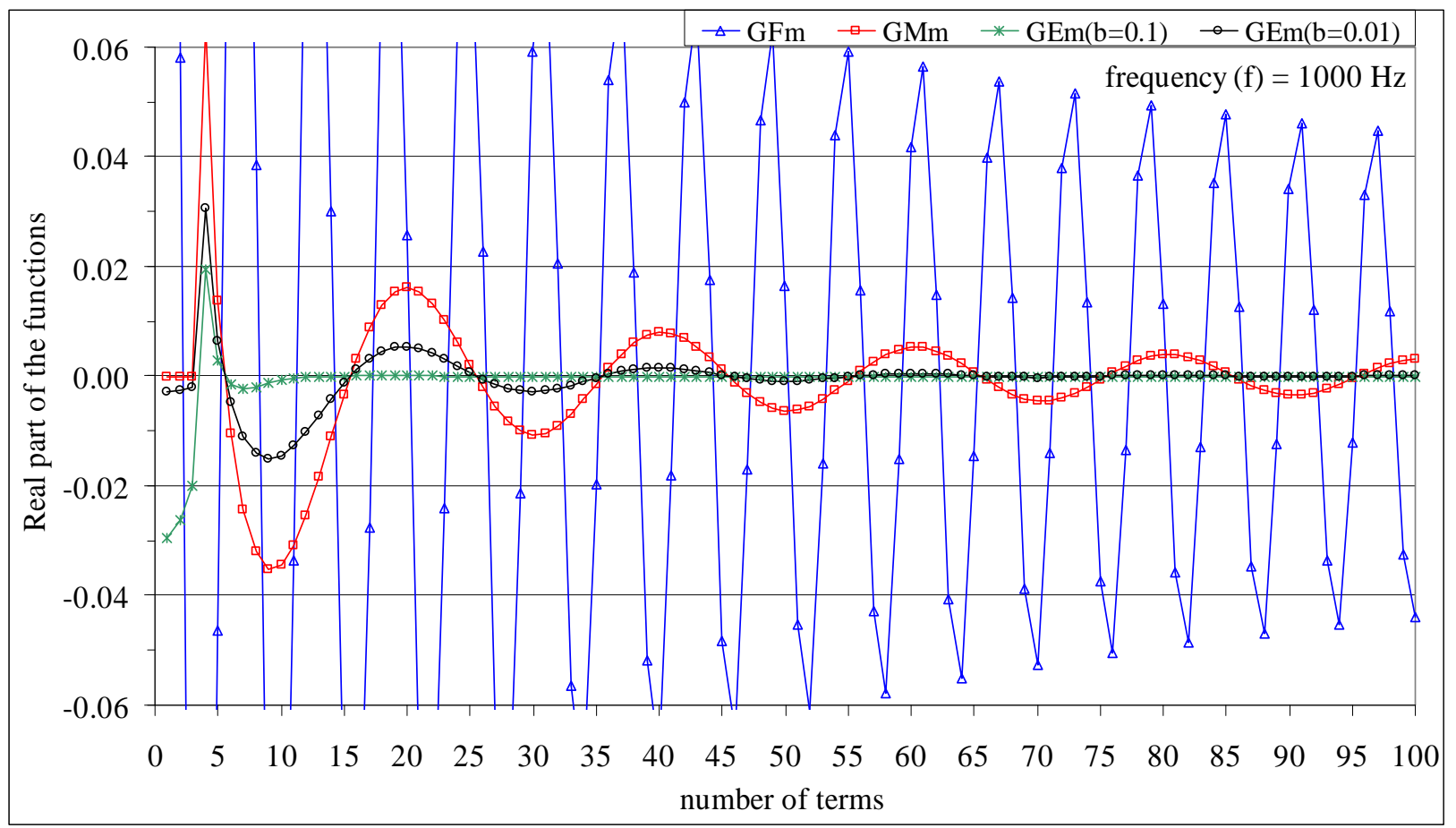

(a) Field point $\mathbf{X}$ close to source point $\mathbf{S}$

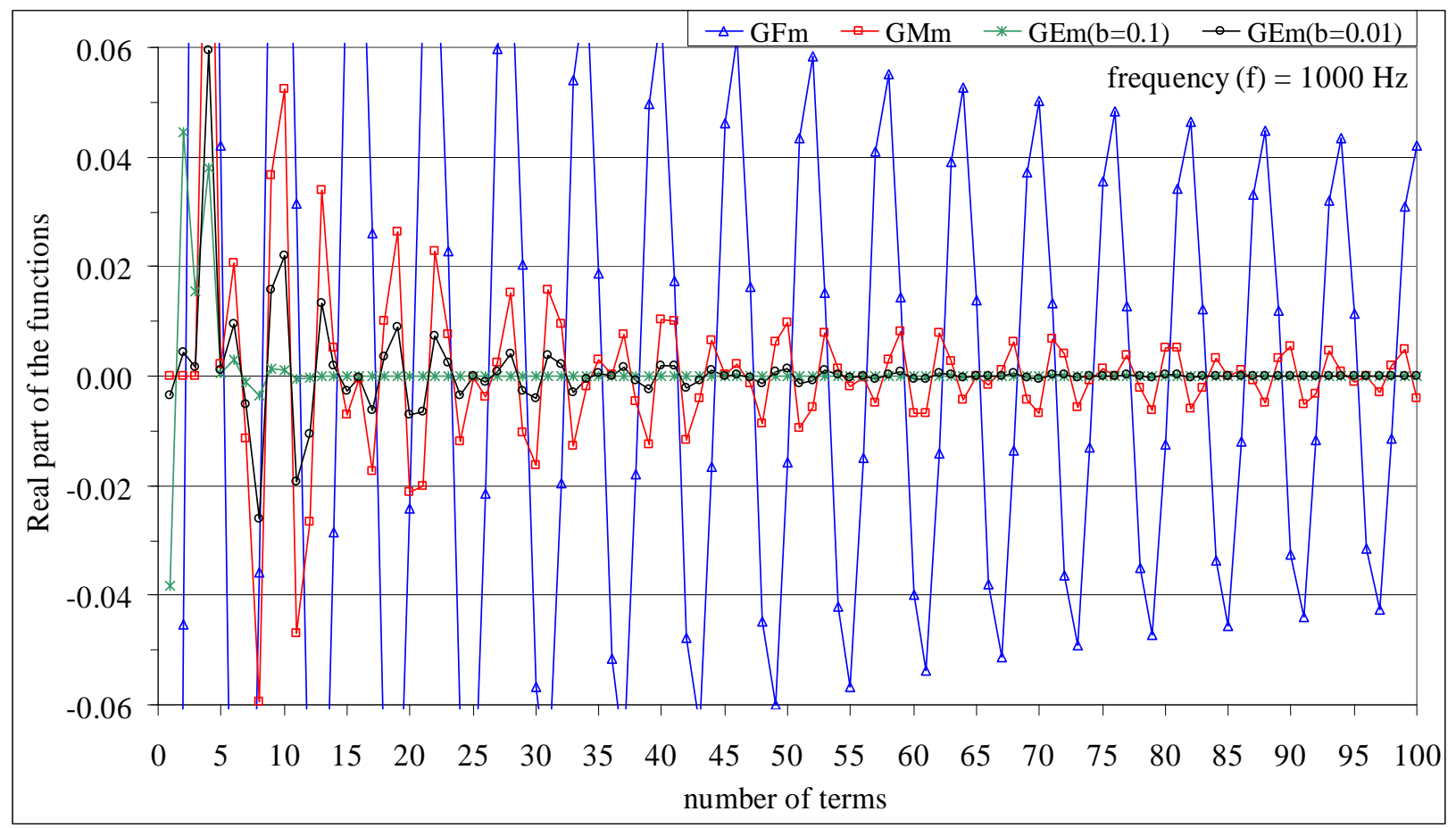

(b) Field point $\mathbf{X}$ far from source point $\mathbf{S}$

Figure 3: Convergence of real part of the summations of $G_{F_{m}}(\mathbf{S}, \mathbf{X}), G_{M_{m}}(\mathbf{S}, \mathbf{X})$ and $G_{E_{m}}(\mathbf{S}, \mathbf{X})$ with number of terms, for $\mathbf{S}$ located at $(1.0,1.1) \mathrm{m}$ and (a) $\mathbf{X}$ located at $(1.0,0.9) \mathrm{m}$ or (b) $\mathbf{X}$ located at $(1 \cdot 0,0.2) \mathrm{m}(h=2 \mathrm{~m})$. 


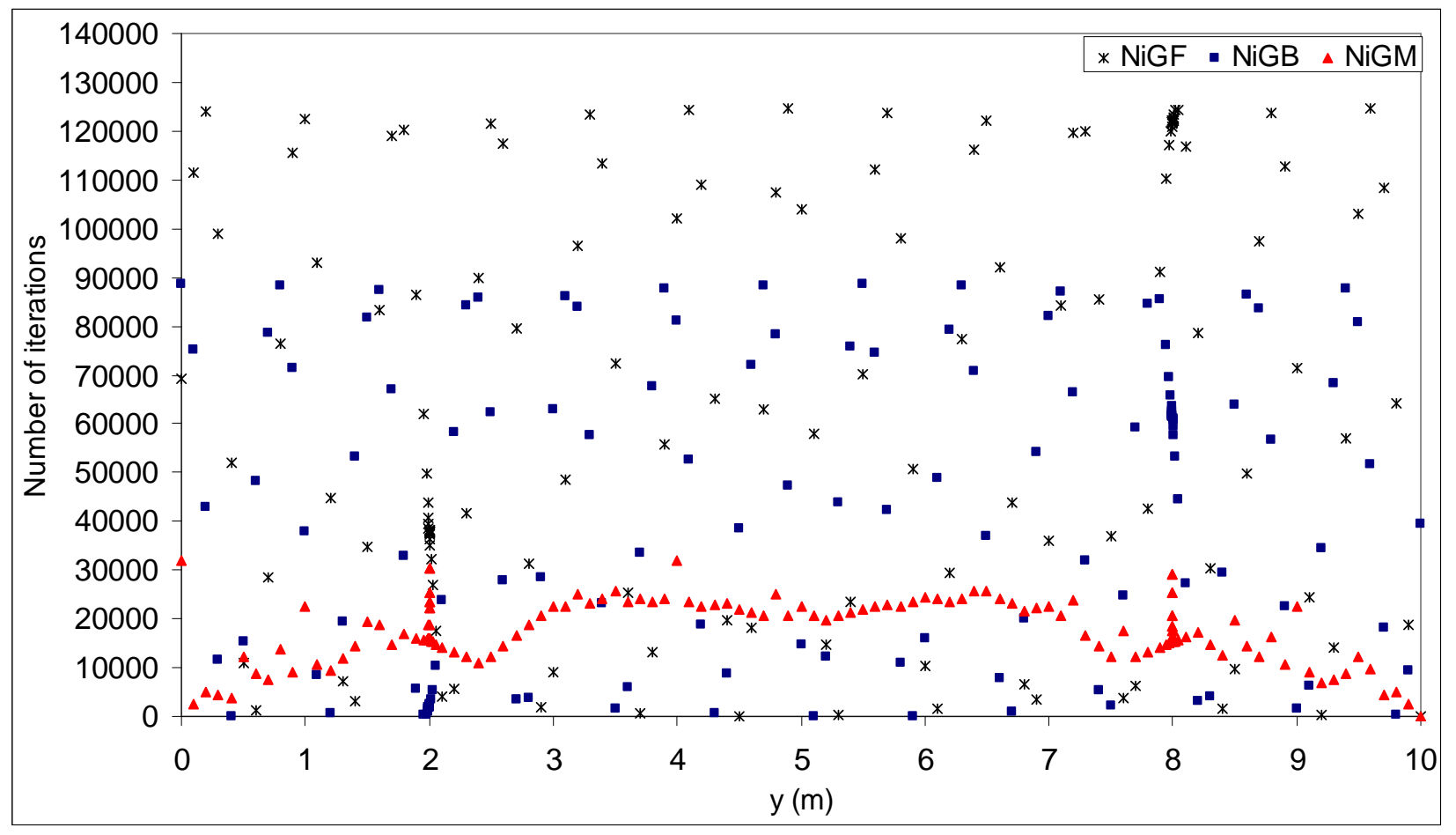

(a) Functions $G_{F}(\mathbf{S}, \mathbf{X}), G_{B}(\mathbf{S}, \mathbf{X})$ and $G_{M}(\mathbf{S}, \mathbf{X})$

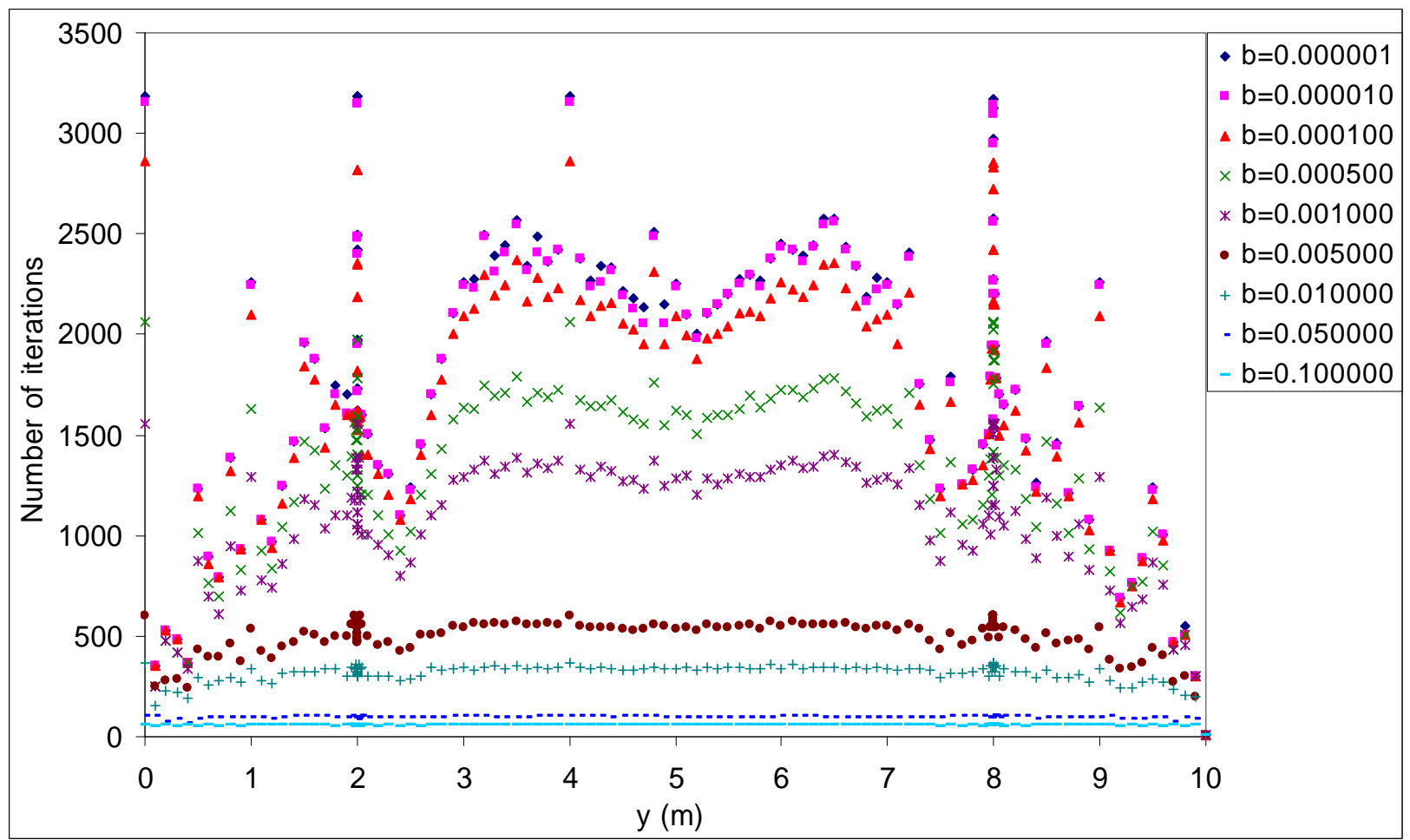

(b) Function $G_{E}(\mathbf{S}, \mathbf{X})$ using nine different values for the parameter $b$

Figure 4: Number of iterations of the functions $G_{F}(\mathbf{S}, \mathbf{X}), G_{B}(\mathbf{S}, \mathbf{X}), G_{M}(\mathbf{S}, \mathbf{X})$ and $G_{E}(\mathbf{S}, \mathbf{X})$ for source point $\mathbf{S}$ located at $(1.0,8.0) \mathrm{m}$ and field points $\mathbf{X}$ located at $x=1.0 \mathrm{~m}, y$ from 0.0 to $10.0 \mathrm{~m}$ 


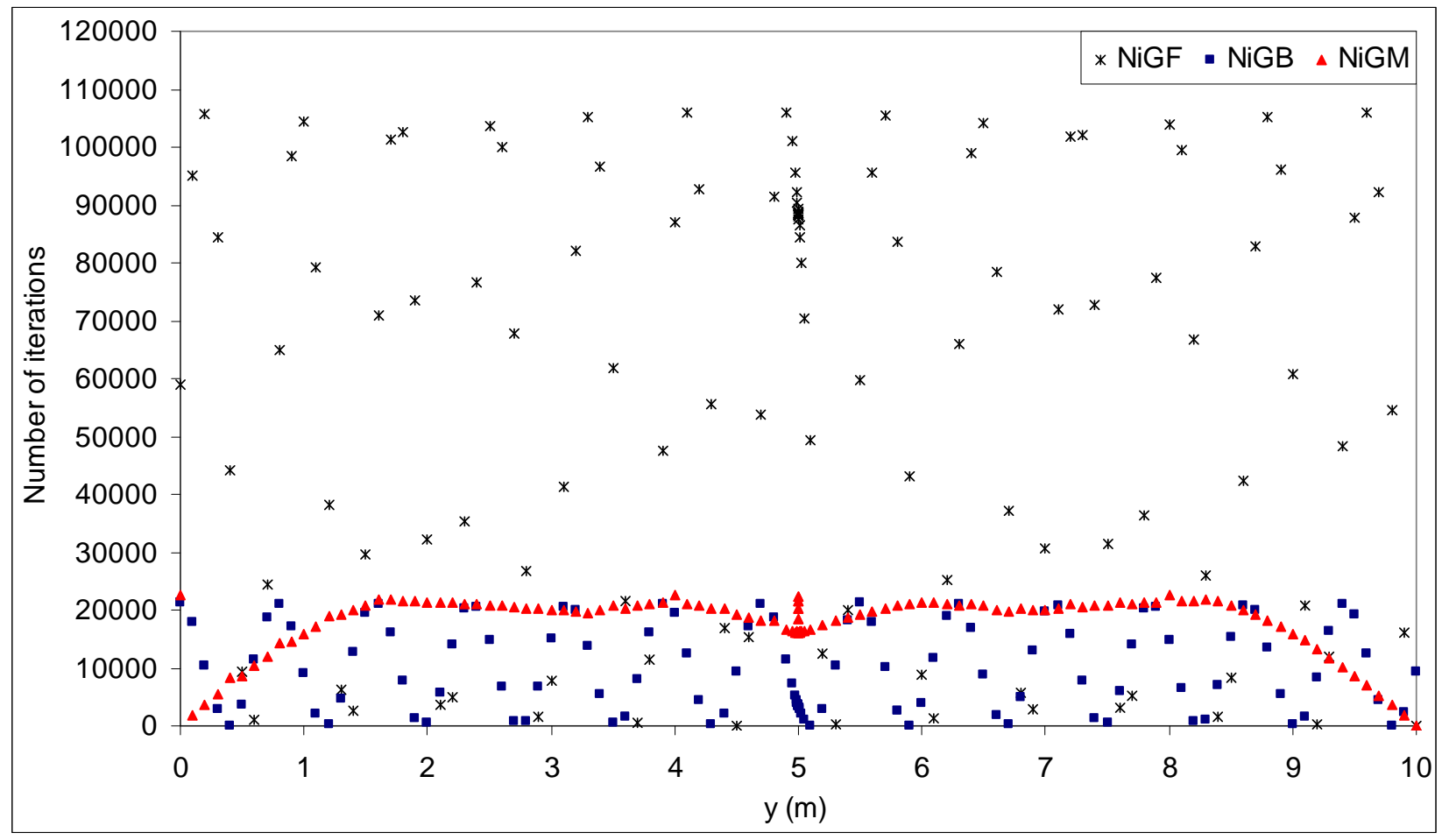

(a) Functions $G_{F}(\mathbf{S}, \mathbf{X}), G_{B}(\mathbf{S}, \mathbf{X})$ and $G_{M}(\mathbf{S}, \mathbf{X})$

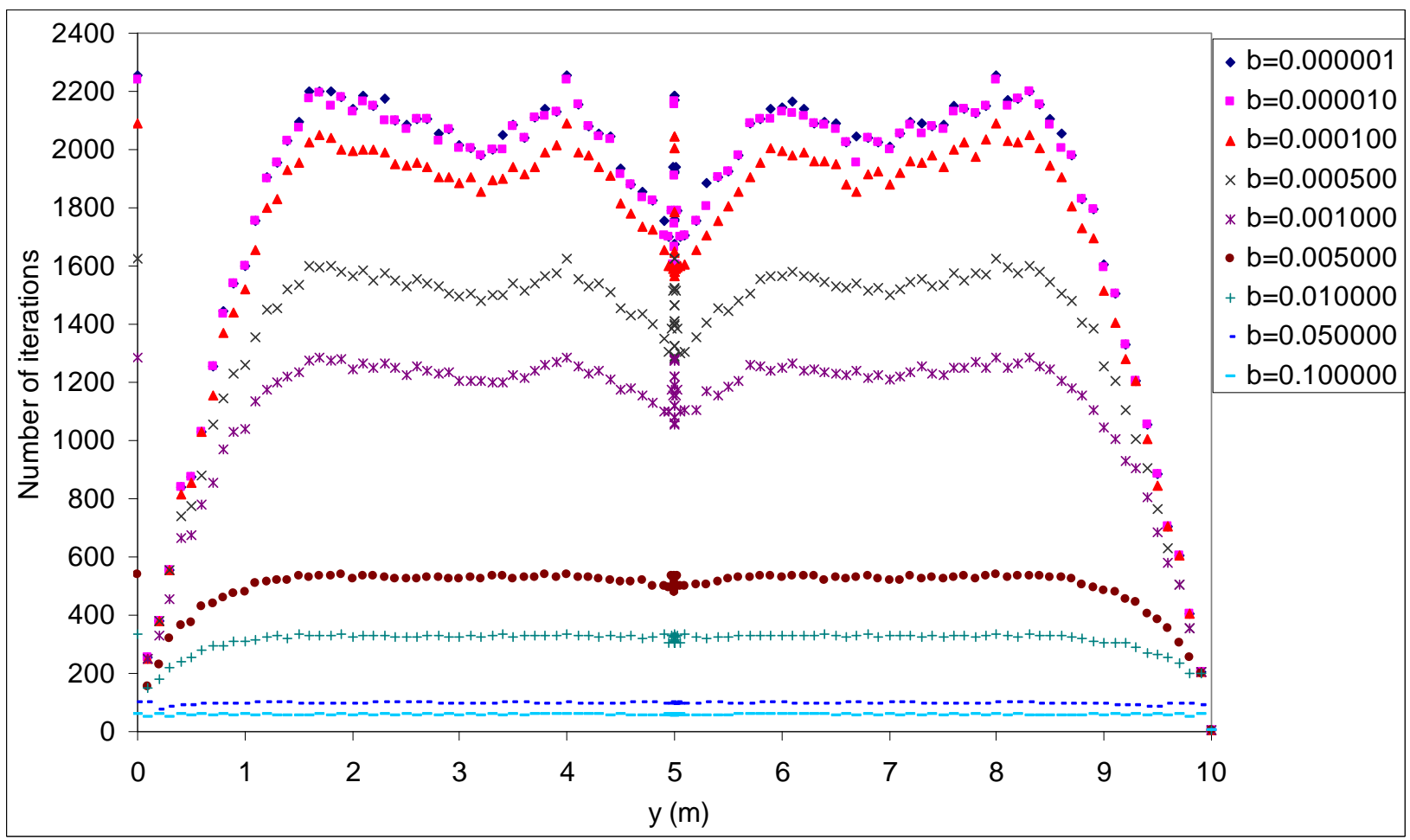

(b) Function $G_{E}(\mathbf{S}, \mathbf{X})$ using nine different values for the parameter $b$

Figure 5: Number of iterations of the functions $G_{F}(\mathbf{S}, \mathbf{X}), G_{B}(\mathbf{S}, \mathbf{X}), G_{M}(\mathbf{S}, \mathbf{X})$ and $G_{E}(\mathbf{S}, \mathbf{X})$ for source point $\mathbf{S}$ located at $(1.0,5.0) \mathrm{m}$ and field points $\mathbf{X}$ located at $x=1.0 \mathrm{~m}, y$ from 0.0 to $10.0 \mathrm{~m}$ 


\section{3- Comparison of the series}

Figures 6 and 7 show the behaviour of the real part of the functions $G_{F}(\mathbf{S}, \mathbf{X}), G_{M}(\mathbf{S}, \mathbf{X})$ and $G_{E}(\mathbf{S}, \mathbf{X})$ in a region of constant depth of $2.0 \mathrm{~m}$. For that, source points were placed at the fixed positions $(7.0,1.3) \mathrm{m}$ and $(3.0,0.5) \mathrm{m}$, while field points were moved along the $x$ - and $y$ direction, respectively. For the latter function, three different values of the parameter $b$ were used.

It is noticed that, for the present case, the real part of the functions $G_{F}(\mathbf{S}, \mathbf{X}), G_{M}(\mathbf{S}, \mathbf{X})$ and $G_{E}(\mathbf{S}, \mathbf{X})$, using $b=0.01$, produced virtually the same results, confirming the validity of Ewald's representation.

In Figure 6, there is a quasi-singularity when the field point is at the position $(7.0,0.8) \mathrm{m}$, while in Figure 7, quasi-singularities occur at positions $(3.1,0.5) m$ and $(3.1,1.5) m$. These quasisingularities are well represented by the function $G_{E}(\mathbf{S}, \mathbf{X})$ with $b=0.01$, but not so for $b=0.1$ or $b=0.2$.

The results obtained with the function $G_{E}(\mathbf{S}, \mathbf{X})$ using different values of parameter $b$, depicted in Figures 6 and 7, are the same when the field points are far from the quasisingularities, except for the case $b=0.2$ in Figure 7 .

Figures 8 and 9 show the behaviour of the real part of the functions $G_{F}(\operatorname{Re}(\mathrm{GF})), G_{B}$ $(\operatorname{Re}(\mathrm{GB})), G_{M}(\operatorname{Re}(\mathrm{GM}))$ and $G_{E}(b)$, using different values for the parameter $b$, for source points located at the fixed positions $(1.0,8.0) \mathrm{m}$ and $(1.0,5.0) \mathrm{m}$, respectively. The field points are located along the vertical line passing through the source point.

There are two singular points in Figure 8, owing to the coincidence of the source and field points at $y=8.0 \mathrm{~m}$, and the parameter $a_{2}$ being equal to zero at $y=2.0 \mathrm{~m}$. Only one singularity is observed in Figure 9, at $y=5.0 \mathrm{~m}$, when the source and field points coincide and the parameter $a_{2}$ is equal to zero since, in the present case, the water depth $Y_{F}$ is equal to $10.0 \mathrm{~m}$.

Figure 10 shows a close-up of the behaviour of the real part of the function $G_{E}(b)$, using nine different values for the parameter $b$, for a source point located at the fixed position $(1.0,5.0)$ $m$ and field point located along the vertical line passing through the source point, from $y=4.0$ to $6.0 \mathrm{~m}$.

The results obtained using all different values of parameter $b$ are the same for values of $y$ lower than $4.7 \mathrm{~m}$ or higher than $5.3 \mathrm{~m}$. Nevertheless, the results close to the singularity clearly improve as the value of parameter $b$ decreases. Hence, the choice of this parameter depends on the position of the source and field points in respect to the singularities of the problem under consideration.

Figure 11 depicts the behaviour of the real part of the functions $G_{F}(\operatorname{Re}(G F)), G_{B}(\operatorname{Re}(G B))$, $G_{M}(\operatorname{Re}(\mathrm{GM}))$ and $G_{E}(b)$, using four different values for the parameter $b$, for a source point located at the fixed position $(2.0,8.0) \mathrm{m}$ and field point located along a different vertical line to that on which the source point is located.

It is noticed that, in this case, the real part of all the functions produced virtually the same results, with the exception of $G_{E}$ using $b=0.8$. 


\section{$\operatorname{Re}(\mathrm{G}(\mathrm{S}, \mathrm{X}))$}

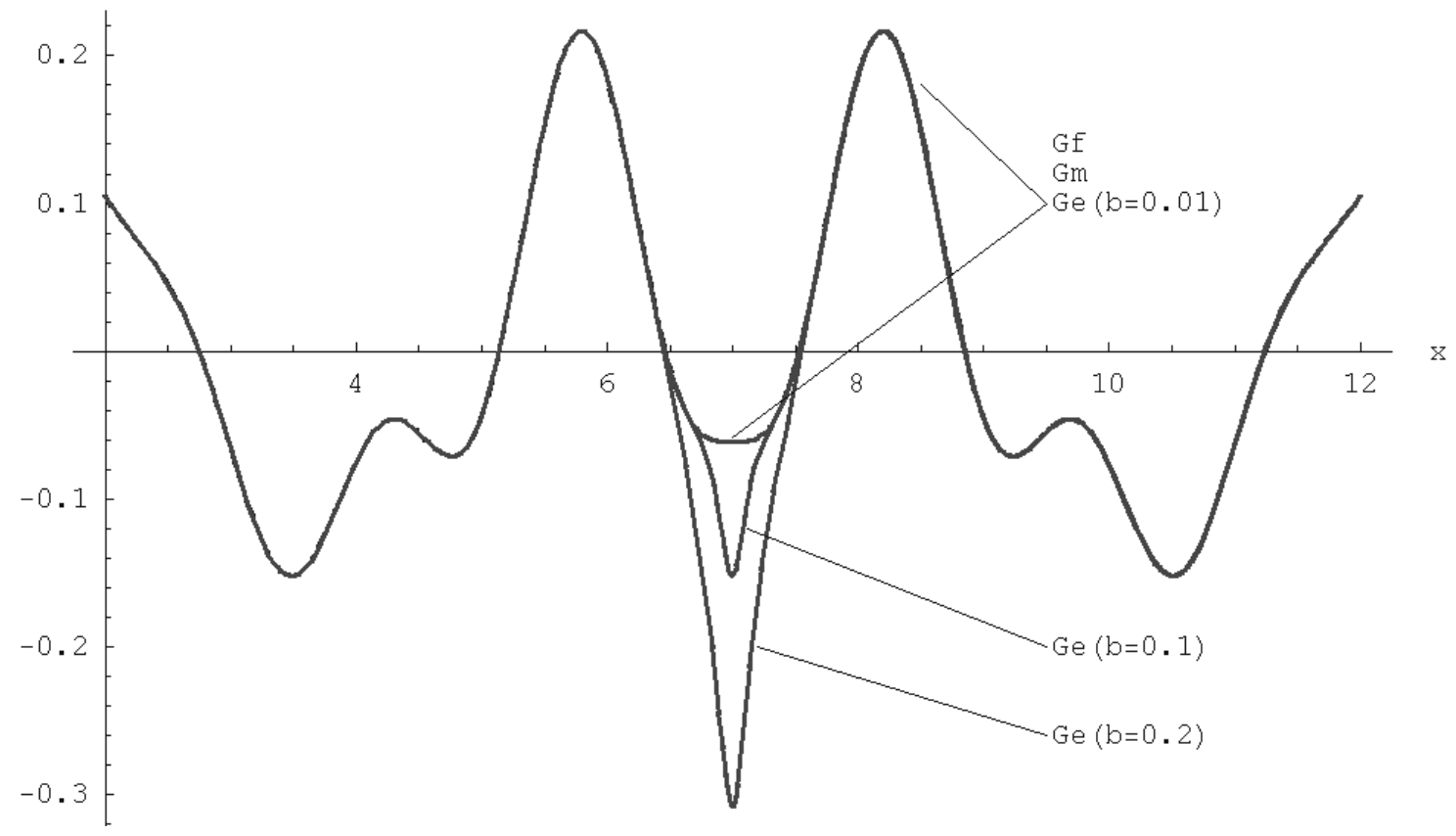

Figure 6: Real part of the functions $G_{F}(\mathbf{S}, \mathbf{X}), G_{M}(\mathbf{S}, \mathbf{X})$ and $G_{E}(\mathbf{S}, \mathbf{X})$ along x-axis for $\mathbf{S}$ located at $(7.0,1.3) \mathrm{m}$ and $\mathbf{X}$ located at $y=0.8 \mathrm{~m}$, X ranging from $2.0 \mathrm{~m}$ to $12.0 \mathrm{~m}(\mathrm{~h}=2 \mathrm{~m})$

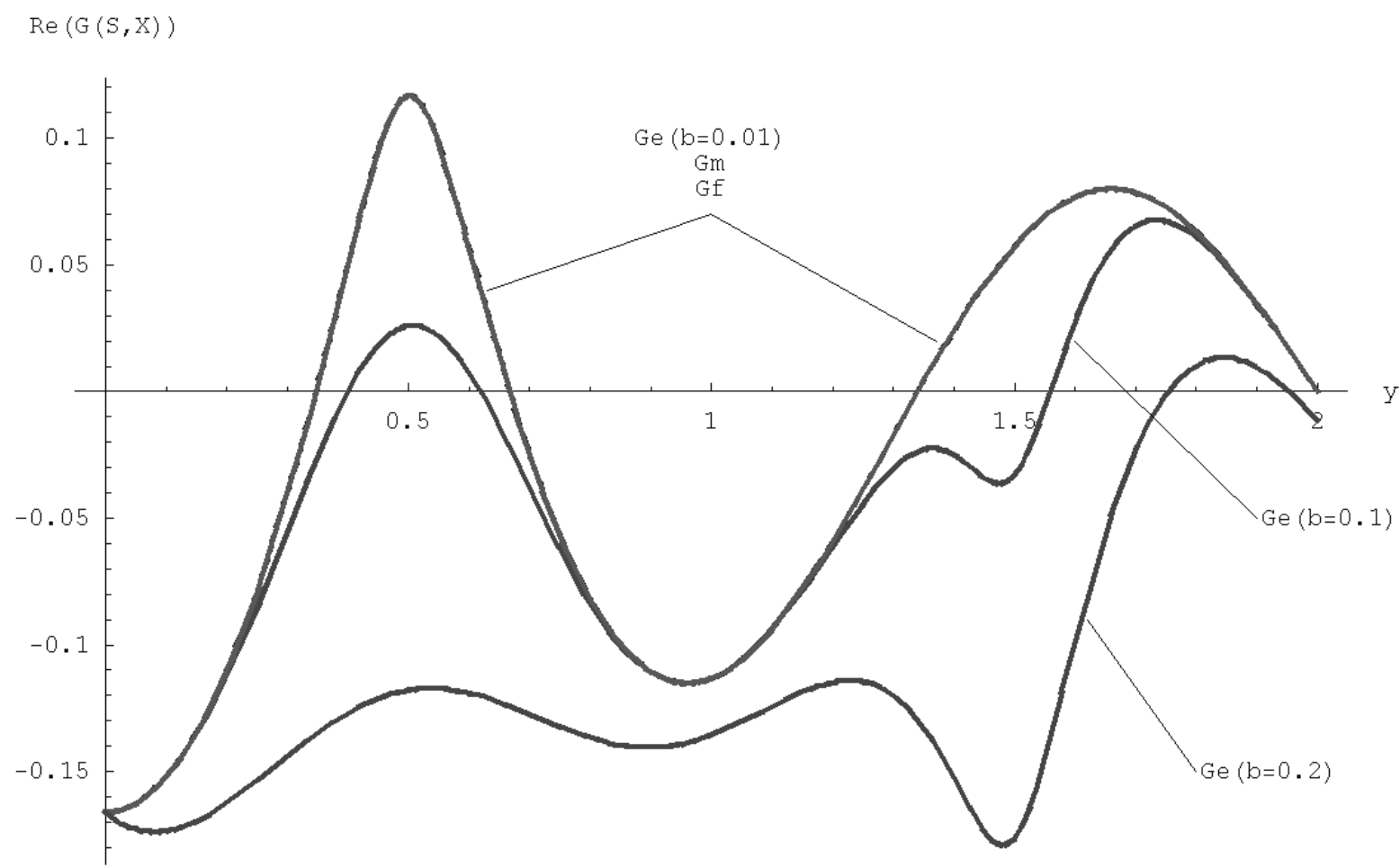

Figure 7: Real part of the functions $G_{F}(\mathbf{S}, \mathbf{X}), G_{M}(\mathbf{S}, \mathbf{X})$ and $G_{E}(\mathbf{S}, \mathbf{X})$ along y-axis for $\mathbf{S}$ located at $(3.0,0.5) \mathrm{m}$ and $\mathbf{X}$ located at $x=3.1 \mathrm{~m}$, y ranging from 0.0 to $2.0 \mathrm{~m}(h=2 \mathrm{~m})$ 


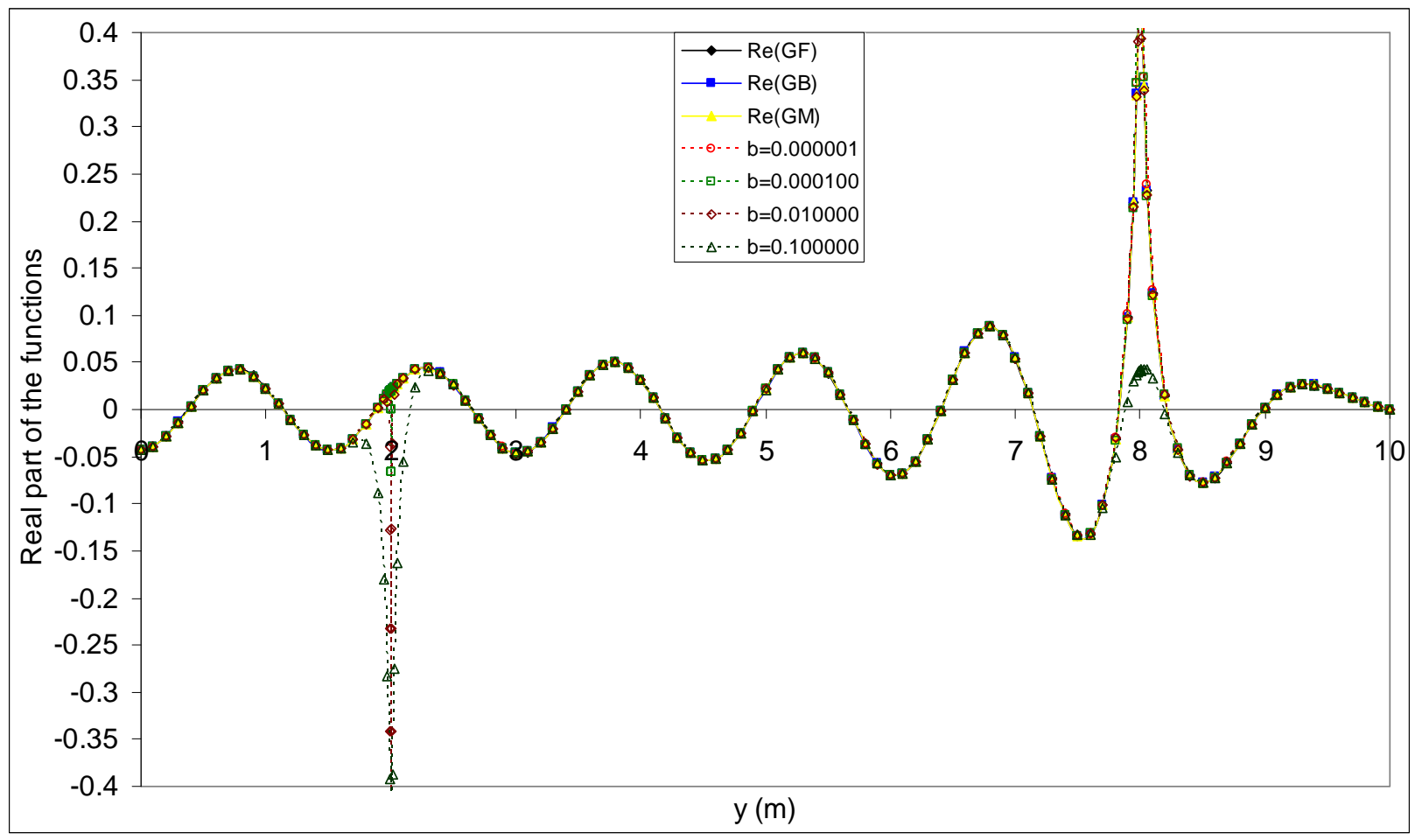

Figure 8: Real part of the functions $G_{F}(\mathbf{S}, \mathbf{X}), G_{B}(\mathbf{S}, \mathbf{X}), G_{M}(\mathbf{S}, \mathbf{X})$ and $G_{E}(\mathbf{S}, \mathbf{X})$ along $y$ coordinate for $\mathbf{S}$ located at $(1.0,8.0) \mathrm{m}$ and $\mathbf{X}$ located at $x=1.0 \mathrm{~m}$, y ranging from 0.0 to $10.0 \mathrm{~m}$

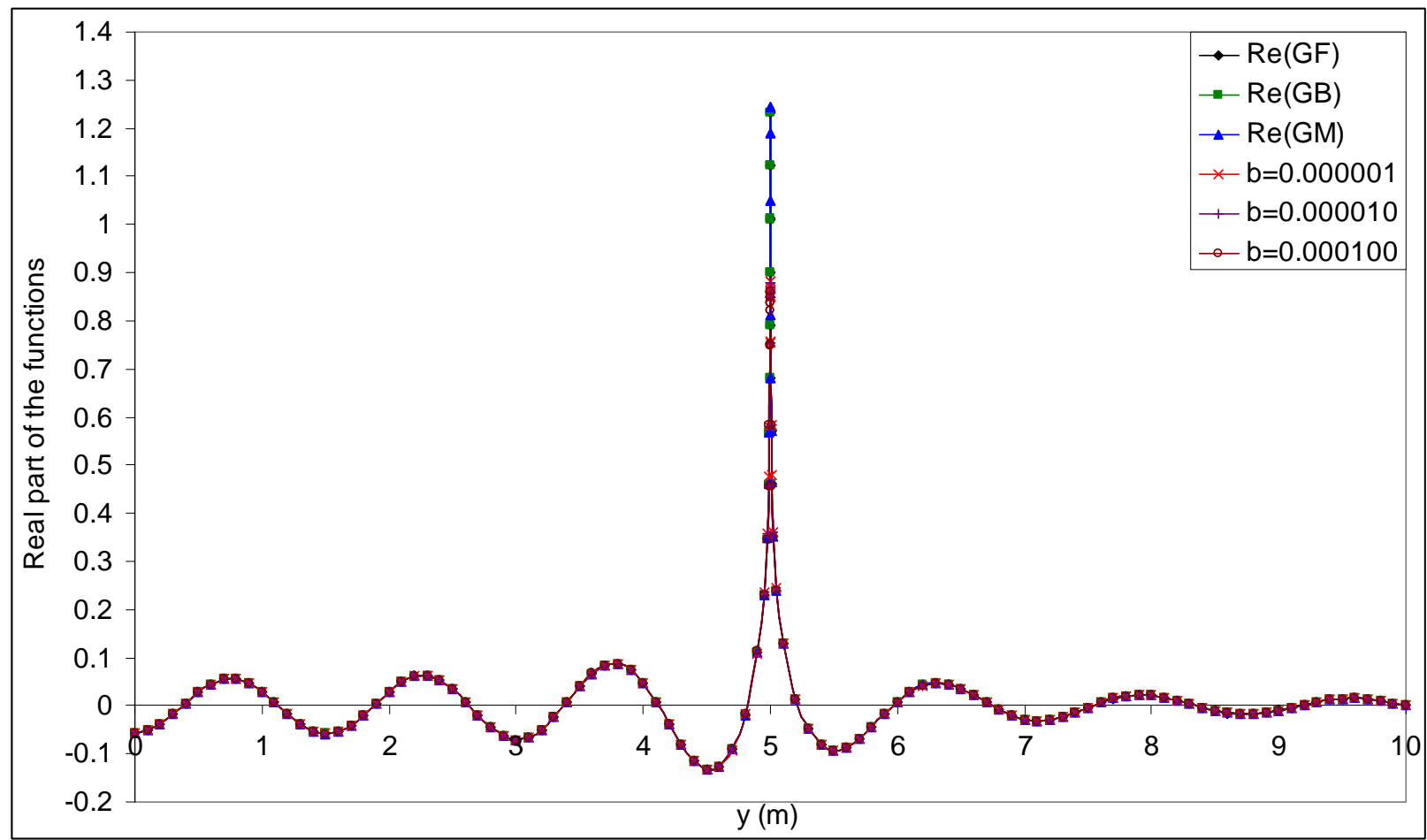

Figure 9: Real part of the functions $G_{F}(\mathbf{S}, \mathbf{X}), G_{B}(\mathbf{S}, \mathbf{X}), G_{M}(\mathbf{S}, \mathbf{X})$ and $G_{E}(\mathbf{S}, \mathbf{X})$ along $y$ coordinate for $\mathbf{S}$ located at $(1.0,5.0) \mathrm{m}$ and $\mathbf{X}$ located at $x=1.0 \mathrm{~m}$, y ranging from 0.0 to $10.0 \mathrm{~m}$ 


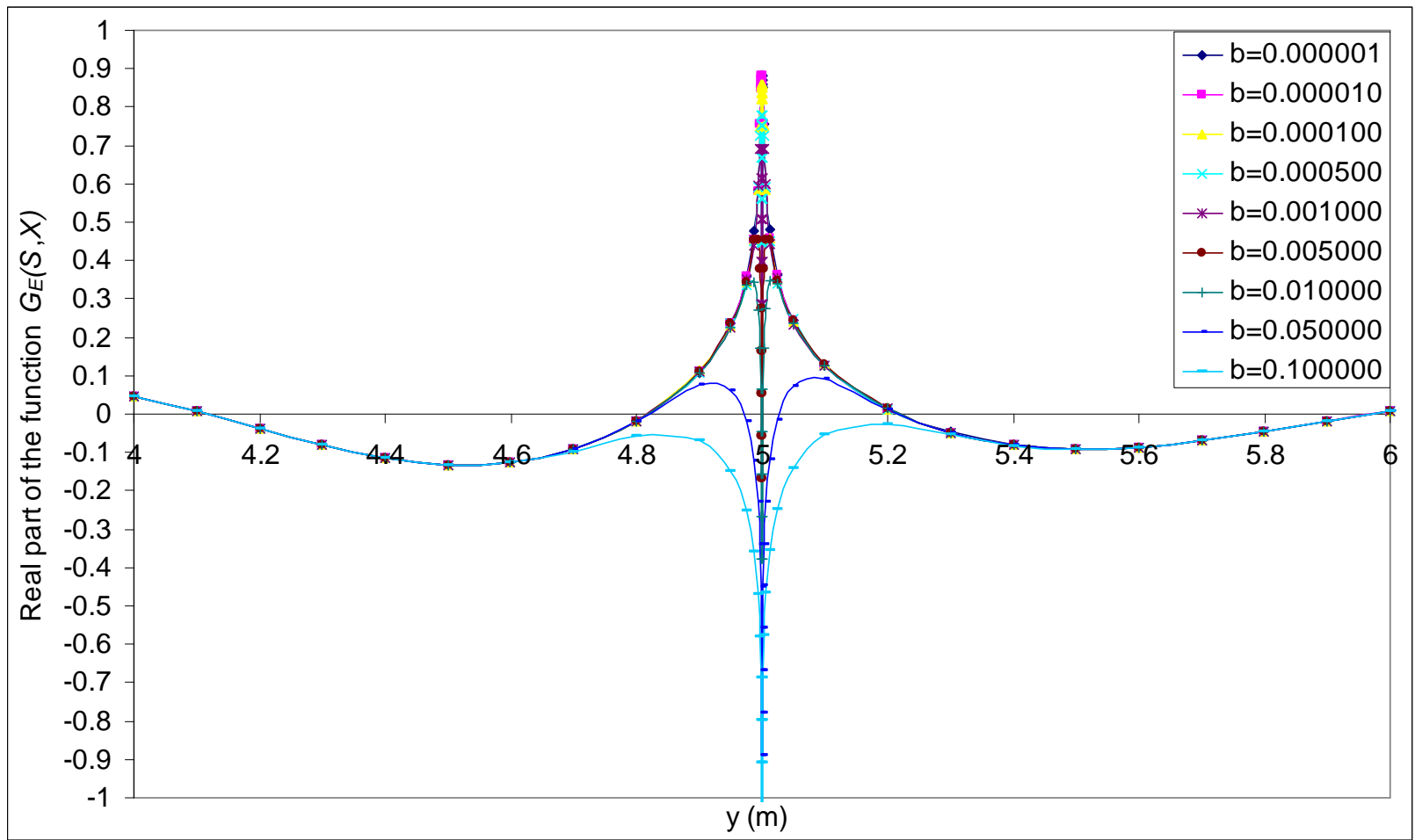

Figure 10: Real part of the functions $G_{E}(\mathbf{S}, \mathbf{X})$ along $y$ coordinate for $\mathbf{S}$ located at $(1.0,5.0) \mathrm{m}$ and $\mathrm{X}$ located at $x=1.0 \mathrm{~m}$, y ranging from 4.0 to $6.0 \mathrm{~m}$

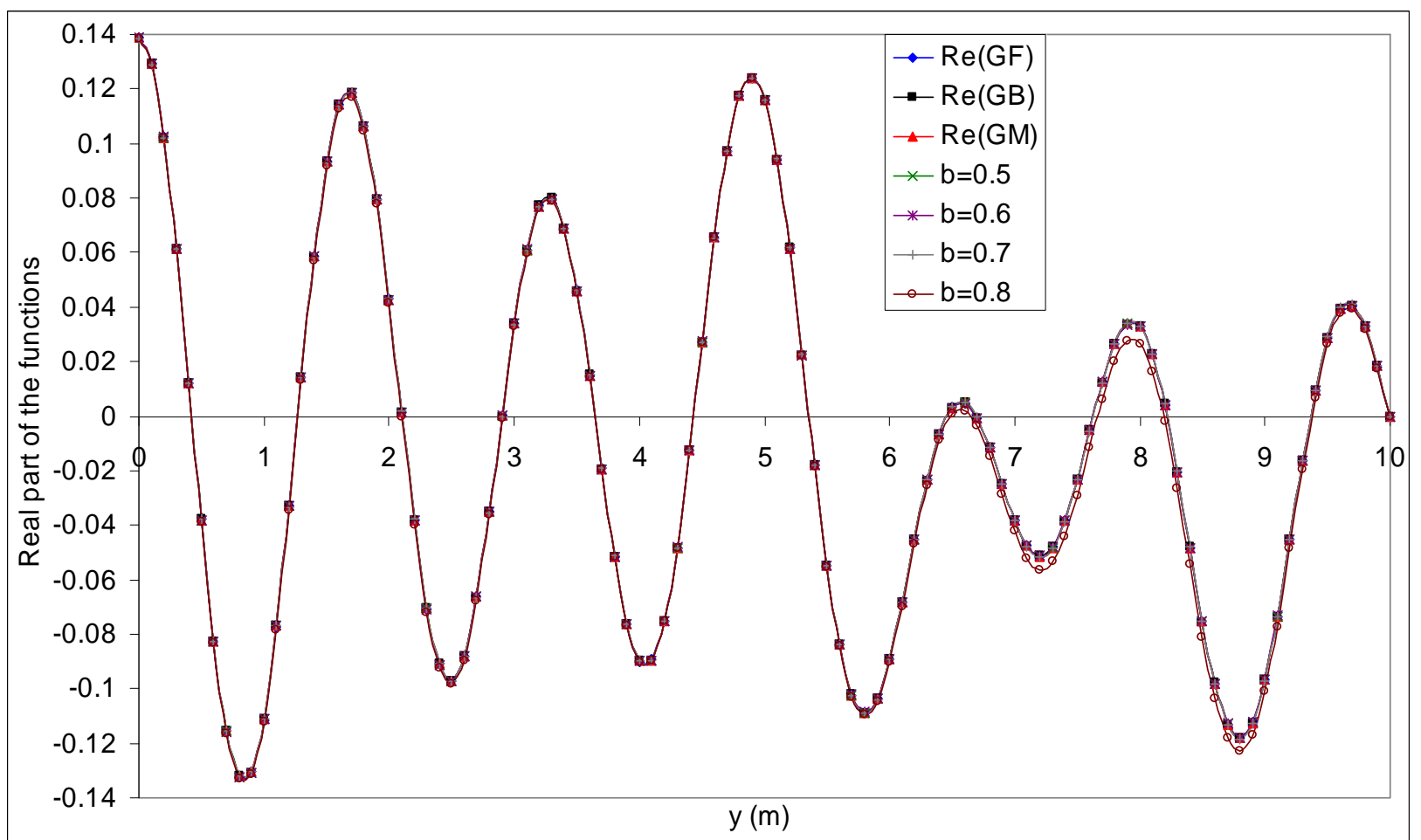

Figure 11: Real part of the functions $G_{F}(\mathbf{S}, \mathbf{X}), G_{B}(\mathbf{S}, \mathbf{X}), G_{M}(\mathbf{S}, \mathbf{X})$ and $G_{E}(\mathbf{S}, \mathbf{X})$ along $y$ coordinate for $\mathbf{S}$ located at $(2.0,8.0) \mathrm{m}$ and $\mathbf{X}$ located at $x=7.0 \mathrm{~m}$, y ranging from 0.0 to $10.0 \mathrm{~m}$ 


\section{Conclusions}

Numerous mathematical techniques exist for accelerating slowly convergent series [7-9]. The Ewald's method, one of the most efficient, was derived and implemented in this paper for speeding-up the calculations of the eigenfunction expansion of the Green's function for underwater acoustics used by the BEM.

It was shown that the Ewald's method is the most accurate and efficient of all the methods implemented, both when the source and field points are located along the same vertical line or otherwise. The influence of the parameter $b$ used to split the infinite integral was investigated, as well as the singular integral generated by the infinite series obtained by the Ewald's method.

Results in Figures 4 and 5 demonstrate that higher values of the parameter $b$ improve the speed of convergence of the series, while Figures 6 to 11 show that accuracy near singularities is improved by using lower values of $b$.

The main conclusion of this preliminary study is that the optimal value of the parameter $b$ in Ewald's method appears to be $b=0.0001$ near singularities and $b=0.1$ away from them. Further studies will be conducted to verify these optimal values and to establish how close to the singularities it is necessary to switch from the higher to the lower value.

\section{References}

[1] F.B. Jensen, W.A. Kuperman, M.B. Porter and H. Schmidt, Computational Ocean Acoustics, American Institute of Physics, USA, 1994.

[2] T.W. Dawson and J.A. Fawcett, A boundary integral equation method for acoustic scattering in a waveguide with nonplanar surfaces, J. Acoust. Soc. Am., 87, 1110-1125, 1990.

[3] T.W. Wu, On computational aspects of the boundary element method for acoustic radiation and scattering in a perfect waveguide, J. Acoust. Soc. Am., 96, 3733-3743, 1994.

[4] J.A.F. Santiago and L.C. Wrobel, 2D modelling of shallow water acoustic wave propagation using subregions technique, Boundary Element Techniques Conference, Queen Mary, University of London, UK, 415-424, 1999.

[5] J.A.F. Santiago and L.C. Wrobel, A boundary element model for underwater acoustics in shallow waters, Computer Modeling in Engineering \& Science, 1, 73-80, 2000.

[6] L. Godinho, A. Tadeu and F. Branco, 3D acoustic scattering from an irregular fluid waveguide via the BEM, Engineering Analysis with Boundary Elements, 25, 443-453, 2001.

[7] C.M. Linton, The Green's function for the two-dimensional Helmholtz equation in periodic domains, Journal of Engineering Mathematics, 33, 377-402, 1998.

[8] C.M. Linton, Rapidly convergent representations for Green's functions for Laplace's equation, Proc. Royal Society A, 455, 1767-1797, 1999.

[9] V. Papanicolaou, Ewald's method revisited: Rapidly convergent series representation of certain Green's functions, J. Comput. Anal. Appl., 1, 105-114, 1999.

[10] P.P. Ewald, Die Berechnung optischer and elektrostatischen Gitterpotentiale, Ann. Phys., 64, 253-268, 1921.

[11] S. Venakides, M.A. Haider and V. Papanicolaou, Boundary integral calculations of twodimensional electromagnetic scattering by photonic crystal Fabry-Perot structures, SIAM J. Appl. Math., 60, 1686-1706, 2000.

[12] L.E. Kinsler, A.R. Frey, A.B. Coppens and J.V. Sanders, Fundamentals of Acoustics, 3rd edition, Wiley, New York, 1982.

[13] L.C. Wrobel and M.H. Aliabadi, The Boundary Element Method, Wiley, Chichester, 2002.

[14] T.K. Pedersen, Modelling Shallow Water Acoustic Wave Propagation, MSc Thesis, University of Rhode Island, USA, 1996.

[15] R. Bellman, A Brief Introduction to Theta Functions, Holt, Rinehart and Winston, Inc., 1961. 